\title{
Genetic Analyses of Soluble Carbohydrate Concentrations in Onion Bulbs
}

\author{
Steven Raines \\ Department of Horticulture, University of Wisconsin, Madison, WI 53706 \\ Cynthia Henson \\ USDA/ARS, Department of Agronomy, University of Wisconsin, Madison, WI 53706
}

Michael J. Havey ${ }^{1}$
USDA/ARS, Department of Horticulture, University of Wisconsin, 1575 Linden Drive,
Madison, WI 53706

\begin{abstract}
Additional Index words. Allium cepa, fructans, reducing sugars, sucrose
Abstract. Fructans are the main soluble carbohydrate in onion (Allium cepa) bulbs and their concentrations show significant correlations with dry weights and pungency. In previous research, we identified regions on chromosomes 5 and 8 associated with higher amounts of soluble carbohydrates in onion bulbs. In this research, we estimated the genetic effects and interactions between these two chromosome regions using larger inbred families grown in field trials over 3 years. Bulbs were evaluated 30 and 90 days after harvest (DAH) for dry weights and soluble carbohydrates. Fructan concentrations decreased significantly between 30 and 90 DAH, consistent with loss of bulb dormancy over this period. Dry weights were negatively correlated with fructose and positively correlated with sucrose and fructans. Analyses of variance and interval mapping revealed that the region on chromosome 5 affected bulb dry weight, whereas the region on chromosome 8 significantly affected both dry weight and fructan concentrations. Regions on chromosomes 5 and 8 showed dominance for increased dry weights and/or soluble solids. Interactions between regions on chromosomes 5 and 8 were significant only for sucrose and the fructan neokestose, indicating that these regions independently contribute to higher amounts of soluble carbohydrates. These results demonstrate that onions with low concentrations of soluble carbohydrates were developed by selecting for relatively few recessively inherited chromosome regions.
\end{abstract}

Dry weights (DWs) of onion bulbs can vary between $6 \%$ and $25 \%$. High DW onions are important for the dehydrating industry to produce onion powders and flakes; low DW or "sweet" onions are preferred by consumers as a fresh vegetable. Soluble solids constitute $\approx 80 \%$ of the DW of onion (Darbyshire and Henry, 1978, 1979, 1981; Shiomi, 1989) and consist mainly of glucose, fructose, sucrose, and fructan polymers (Darbyshire and Henry, 1978; Shiomi, 1989). These fructans are an important dietary source of fiber that resists digestion (Moshfegh et al., 1999). As a prebiotic, fructans stimulate the growth of beneficial Bifidobacteria bacteria in the colon (Roberfroid, 2005).

In onion, fructans are synthesized in the vacuole by fructosyltransferases, which attach fructose molecules to fructose moieties on sucrose or fructans with degrees of polymerization (DP) of fructoses from 3 to 12 (Vijn et al., 1997). Initially, 1-kestose (DP = 3) is synthesized by the enzyme sucrose:sucrose 1-fructosyltransferase, which removes fructose from sucrose and attaches it to a second sucrose forming the

\footnotetext{
Received for publication 29 June 2009. Accepted for publication 19 Oct. 2009. This work was supported by a Biotech Training Fellowship to SR and the Agricultural Research Service of the USDA.

We gratefully acknowledge that high-performance liquid chromatography runs were completed by Mr. Charles Karpelenia in the Henson laboratory. We thank the Kincaid farm, Palmyra WI, for field space.

Names are necessary to report factually on available data; however, the U.S. Department of Agriculture (USDA) neither guarantees nor warrants the standard of the product, and the use of the name by USDA implies no approval of the product to the exclusion of others that may also be suitable.

${ }^{1}$ Corresponding author. E-mail: mjhavey@wisc.edu.
}

first fructan in the inulin series (linear chain fructans) and releasing glucose. Alternatively, fructan:fructan 6G-fructosyltransferase (6G-FFT) attaches fructose to the sixth carbon of the glucose residue of sucrose forming a trisaccharide, neokestose $(\mathrm{DP}=3)$ with a $\beta(2,6)$ linkage using 1 -kestose as a fructose donor. Neokestose is the first fructan of the inulin neoseries (branched chain fructans) and can be elongated from both fructose residues with $\beta(2,1)$-linked fructose units (Ritsema and Smeekens, 2003). Fructans with longer DP are polymerized by fructan:fructan 1-fructosyltransferase (1-FFT) activity. 1-FFT has not been found in onion; however 6G-FFT also shows 1FFT activity to produce the inulin series and neoseries molecules (Ritsema et al., 2003).

Two classes of enzymes, acid invertases and fructan exohydrolases, are involved in the degradation of fructans. Acid invertase is found in the vacuole and normally functions in the irreversible breakdown of sucrose into glucose and fructose, but it also has the ability to breakdown 1-kestose (Ritsema and Smeekens, 2003). Fructan exohydrolases (FEHs) are also localized in the vacuoles and cleave terminal fructose residues. No FEHs have been cloned from onion, but 1-FEH activity during storage has been characterized (Benkeblia et al., 2005).

Genetic studies of soluble carbohydrates in onion bulbs have revealed significant phenotypic and genotypic correlations among soluble solids content, DW, and pungency (Galmarini et al., 2001; Lin et al., 1995; Simon, 1995; Sinclair et al., 1995; Suzuki and Cutcliffe, 1989). One region on chromosome 5 accounted for significant amounts of variation for these traits, showing positive additive effects of alleles from the high DW parent (Galmarini et al., 2001). However, this region showed no 
significant effects on these traits when the family means were adjusted to a mean DW, suggesting that this region may control the relative water content of bulbs (Havey et al., 2004). This region could affect cell sizes or densities; Kahane et al. (2001) proposed that environmental signals such as the ratio of red to far-red light play an important role in the ability of onion to increase DW and soluble solids concentrations. McCallum et al. (2006) observed strong negative correlations between reducing sugars and fructans and identified a major quantitative trait locus (QTL) on chromosome 8 for bulb carbohydrate content. The significant effects of these two chromosome regions were supported by assignment of major genes controlling bulb carbohydrate contents to chromosomes 5 and 8 (Hang et al., 2004; Masuzaki et al., 2007). The purpose of this research was to estimate the genetic effects and interactions of regions on chromosomes 5 and 8 on DW and soluble carbohydrates using two inbred families segregating across these regions.

\section{Materials and Methods}

Plant materials. Segregating families were derived from a cross between inbreds 'Brigham Yellow Globe' (BYG) 15-23 and 'Ailsa Craig' (AC) 43, which possess relatively high and low amounts of soluble solids, respectively (Galmarini et al., 2001). A single $F_{1}$ plant was self-pollinated to produce the $F_{2}$ family and individual $\mathrm{F}_{2}$ plants were self-pollinated to produce $\mathrm{F}_{3}$ families. At least 10 random $\mathrm{F}_{3}$ plants from each family were intercrossed in cages to produce $\mathrm{F}_{3} \mathrm{M}$ (massed) families with sufficient seed for field production of bulbs, analysis of soluble solids (Galmarini et al., 2001; Havey et al., 2004), and mapping of molecular markers (King et al., 1998; Martin et al., 2005). For this study, two $\mathrm{F}_{3} \mathrm{M}$ families (16278 and 16292) were chosen because they were heterozygous across regions on chromosomes 5 and 8 significantly associated with soluble solids contents (Galmarini et al., 2001). Random $\mathrm{F}_{3} \mathrm{M}$ progenies were self-pollinated to produce the $\mathrm{F}_{3} \mathrm{MS}$ families. At least 10 bulbs from each of these $\mathrm{F}_{3} \mathrm{MS}$ families were intercrossed to produce the $\mathrm{F}_{3} \mathrm{MSM}$ families with adequate seed for field production of bulbs. For $72 \mathrm{~F}_{3} \mathrm{MSM}$ families (31 from 16278 and 41 from 16292), bulbs were produced in commercial fields near Palmyra, WI, in each of 3 years (2004 to 2006) in a randomized complete block design with three replications under normal production conditions. Bulbs were harvested in September of each year and stored in the dark at $\approx 5{ }^{\circ} \mathrm{C}$. Ten randomly selected unsprouted bulbs from each family were sampled $\approx 30$ and $90 \mathrm{~d}$ after harvest (DAH). According to Randle (1992), three replications of 10 -bulb samples were adequate to detect differences of $0.77 \%$ in soluble carbohydrates and $0.11 \%$ in DW; this sample size has also been used in our previous studies (Galmarini et al., 2001). After removing the dry outer scales, the bulbs were cut in half longitudinally. One of the halves was used to measure wet and dry weights. The other half was cut into quarters. One quarter from each of the 10 bulbs was juiced together using a Champion juicer (Plastaket Manufacturing Co., Lodi, CA). Ten milliliters of juice was immediately place in boiling water for $5 \mathrm{~min}$ to stop enzyme activity and then stored at $-20{ }^{\circ} \mathrm{C}$. Frozen samples were thawed and centrifuged at 6000 $g_{\mathrm{n}}$ for $10 \mathrm{~min}$ to pellet large debris. One milliliter of supernatant was transferred to a microcentrifuge tube and centrifuged for 5 min at $13,000 g_{n}$ to pellet smaller debris. Juice was filtered through 0.20- $\mu \mathrm{m}$ nylon membranes (Millipore, Bedford, MA) and diluted 1:100 in double-distilled water with $0.1 \%$ sodium azide to prevent microbial contamination. One milliliter of diluted juice was transferred to a high-performance liquid chromatography (HPLC) vial and stored at $4{ }^{\circ} \mathrm{C}$.

High-PERFormance LiQUid CHROMATOGRAPHY RUNS. Carbohydrates were separated on CarboPac PA-1 column $(4 \times 250$ $\mathrm{mm})$ with a CarboPac PA-1 guard column $(4 \times 50 \mathrm{~mm}$; Dionex, Sunnyvale, CA) using HPLC (VP series; Shimadzu, Kyoto, Japan). The elution gradient was from 99.5\% eluant A (100 mM $\mathrm{NaOH})$ and $0.5 \%$ eluant $\mathrm{B}(100 \mathrm{~mm} \mathrm{NaOH}$ in $600 \mathrm{~mm}$ sodium acetate) to $43 \% \mathrm{~A}: 57 \% \mathrm{~B}$ over $20 \mathrm{~min}$ followed by a $10-\mathrm{min}$ wash with $99.5 \%$ A: $0.5 \%$ B. Glucose, fructose, and sucrose were identified by comparing peak retention times with those of authentic standards and were quantified using a response curve generated with three concentrations of each standard. Standards of 1-kestose, $(1,1)$ nystose, and $(6 \mathrm{G}, 1)$ nystose were used to establish retention times. The retention time of neokestose was established in previous work using similar methods (Benkeblia et al., 2005, 2007; Henson and Livingston, 1996; Shiomi et al., 1997). The contents of fructans for which there were no standard curves are expressed as chromatogram peak areas.

GENETIC MARKERS. Segregations were scored for molecular markers API92, AOB236, API66, and API47 on chromosome 5 and ACM033, ACABE58, and AJB72 on chromosome 8. These markers are associated with major QTL affecting soluble carbohydrate concentrations in onion (Galmarini et al., 2001; Havey et al., 2004). Genomic DNA from each $\mathrm{F}_{3} \mathrm{MS}$ family was isolated from seedlings using CTAB extractions and purified by $\mathrm{CsCl}$ centrifugation (Bark and Havey, 1995). Restriction fragment length polymorphism makers were scored as described by King et al. (1998), microsatellites as described by Jakse et al. (2005), and single-nucleotide polymorphisms as described by Martin et al. (2005).

Statistical analysis. Normality of the untransformed residuals was checked by the Shapiro-Wilk test using SAS (Version 9.1 for Windows; SAS Institute, Cary, NC) PROC UNIVARIATE function. Analyses of variance (ANOVAs) were performed using the PROC MIXED function. Year and block within year were treated as random variables and all other variables were considered fixed. Least square means were calculated for juicing date (30 and $90 \mathrm{DAH}$ ), family background (16278 or 16292), and markers for all traits. Significance of differences between means was determined using the protected least-significant difference test. Percent dry weight was calculated by dry weight over fresh weight multiplied $\times 100$. Fructose, glucose, and sucrose concentrations are reported as $\mu \mathrm{g} / 20 \mu \mathrm{L}$. As a result of lack of sufficient standards for quantification, 1-kestose, neokestose, and total fructan are reported as HPLC peak areas $\times 10^{5}$.

Analyses of QTL were performed using least square means and composite interval mapping of Windows QTL Cartographer Version 2.5 (Wang et al., 2000). The model used unlinked markers as controls. QTL thresholds for each trait were determined by permutation tests conducted 1000 times at a 0.05 significance level (Churchill and Doerge, 1994). QTLs with logarithm of odds scores above the threshold were considered significant. The percentage of phenotypic variance $\left(R^{2}\right)$ explained and the additive and dominance effects of QTLs were estimated at the highest likelihood ratio graph peak using Windows QTL Cartographer.

\section{Results and Discussion}

Segregation of MARKers. Genetic markers API66, API92, AOB236, ACM033, and ACABE58 fit $(P>0.05)$ the $1: 2: 1$ 
segregation ratio for codominant markers. API47 and AJB72 fit the 3:1 segregation ratio for dominant markers (data not shown). These results demonstrate that both chromosome regions segregated normally in this study.

MaIN EFFECTS FROM ANALYSES OF VARIANCE. Onion bulbs possess significant concentrations of soluble carbohydrates, primarily reducing sugars (fructose and glucose), sucrose, and fructans. Concentrations of specific carbohydrates change during storage, attributable primarily to dehydration and the activities of fructan exohydrolases as the onion bulb emerges from dormancy (Benkeblia et al., 2005). Single-factor ANOVAs for genetic markers on chromosomes 5 and 8 revealed significance for at least one of the evaluated traits with total fructans and DW consistently significant across all markers (data not shown). Markers API92 and ACM033 showed the highest significance levels for the most traits and were chosen for analysis with year, juicing date, family background, and their interactions. In the two-marker analyses, years were highly significant (Table 1), consistent with previous multiyear investigations of carbohydrate concentrations in onion (Galmarini et al., 2001; Kahane et al., 2001). Juicing date was highly significant $(P<0.001)$ for all traits except fructose and sucrose (Table 1). Concentrations of 1-kestose, neokestose, and fructans significantly decreased between 30 and 90 DAH (Table 2) consistent with loss of bulb dormancy over this period (Benkeblia et al., 2004, 2005, 2007; Jaime et al., 2001; Yasin and Bufler, 2007). The activity of fructan exohydrolase generally peaks between 8 and 12 weeks after harvest, hydrolyzing fructans to sucrose and reducing sugars (Benkeblia et al., 2005).

Family background was highly significant $(P<0.01)$ for all traits except fructose (Table 1). The 16278 background had higher reducing sugars and significantly lower DW, sucrose, and fructans than the 16292 background (Table 2). The significance of the family background is consistent with genotypes across a region on chromosome 3 previously shown to affect DW and soluble solids (Galmarini et al., 2001; Havey et al., 2004; McCallum et al., 2006). Family 16278 was homozygous for the low-solids region from AC43 and family 16292 was homozygous for the high-solids region from BYG15-23 on chromosome 3 .

Table 1. Significant sources of variance for the two-marker (ACM033 and API92) analyses of dry weight (DW) and soluble carbohydrates in onion bulbs.

\begin{tabular}{|c|c|c|c|c|c|c|c|}
\hline \multirow[b]{3}{*}{ Source of variation ${ }^{z}$} & \multicolumn{7}{|c|}{ Significance $^{\mathrm{y}}$} \\
\hline & \multirow[b]{2}{*}{$\mathrm{DW}^{\mathrm{x}}$} & \multicolumn{6}{|c|}{ Soluble carbohydrates } \\
\hline & & Fructose & Glucose & Sucrose & 1-Kestose & Neokestose & Fructan \\
\hline Year & $* * *$ & $* * *$ & $* * *$ & $* * *$ & $* * *$ & $* * *$ & $* * *$ \\
\hline Juicing & $* * *$ & NS & $* * *$ & NS & $* * *$ & $* * *$ & $* * *$ \\
\hline Background & $* * *$ & NS & $* *$ & $* * *$ & $* * *$ & $* * *$ & $* * *$ \\
\hline ACM033 & NS & NS & $* *$ & NS & $* *$ & $* * *$ & $* * *$ \\
\hline API92 & $* * *$ & $* * *$ & $*$ & NS & $* * *$ & $* * *$ & $* * *$ \\
\hline Juicing $\times$ Background & NS & NS & NS & NS & $* * *$ & $* * *$ & $* * *$ \\
\hline Background $\times$ ACM033 & NS & NS & NS & NS & $* *$ & $* * *$ & $* * *$ \\
\hline Background $\times$ API92 & NS & NS & NS & NS & NS & $* *$ & $*$ \\
\hline ACM033 × API92 & $* *$ & NS & NS & NS & NS & $* *$ & NS \\
\hline Background $\times$ ACM033 × API92 & NS & NS & NS & $*$ & $* * *$ & $* * *$ & $* *$ \\
\hline
\end{tabular}

Zuicing of onion bulbs occurred at 30 and $90 \mathrm{~d}$ after harvest; background refers to families 16278 and 16292.

$\mathrm{y}_{\mathrm{NS}}, *, * * * *$, NS represent nonsignificant and significant at $P<0.05,0.01$, and 0.001 , respectively, from analysis of variance.

${ }^{\mathrm{x}} \mathrm{DW}=$ bulb dry weight.

Table 2. Least-square means for percent dry weight (DW) and amounts of soluble carbohydrates in onion bulbs for genetic backgrounds and chromosome regions from parents BYG15-23 and AC43 at markers API92 and ACM033 at 30 and $90 \mathrm{~d}$ after harvest (DAH).

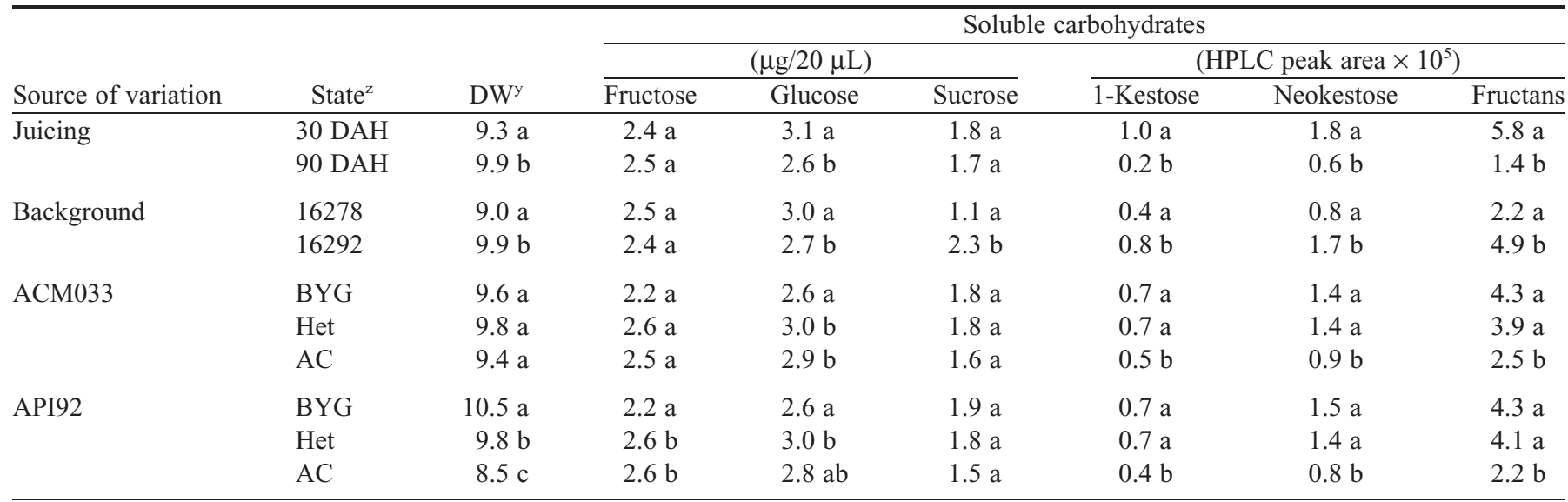

zJuicing of onion bulbs occurred at 30 and 90 DAH. Genetic backgrounds were 16278 and 16292. BYG and AC refer to homozygotes for parental alleles at marker loci (ACM033 and API92). Het designates heterozygotes.

${ }^{\mathrm{y}}$ Least square means followed by the same letter were not significantly different $(P<0.05)$ using the protected least significant difference test. HPLC $=$ high-performance liquid chromatography. 
Genotypes at API92 on chromosome 5 were significantly associated with DW, fructose, glucose, and fructans (Table 1). The BYG15-23 allele at API92 increased DW and fructans as well as decreased amounts of reducing sugars relative to the AC43 allele (Table 2). Genotypes at ACM033 on chromosome 8 were significantly associated with glucose and fructans, but not DW, fructose, or sucrose (Table 2). The BYG15-23 allele at ACM033 increased fructan concentrations and decreased amounts of reducing sugars relative to the AC43 allele (Table 2).

INTERACTIONS FROM ANALYSES OF VARIANCE. The interaction between markers ACM033 and API92 was significant $(P<$ 0.05 ) only for DW and neokestose, indicating that the regions on chromosomes 5 and 8 independently contributed to higher sucrose and fructan contents. Family background interacted significantly with juicing date, API92, and ACM033 for fructan concentrations, but not for DW, reducing sugars, or sucrose (Table 1). For all of these interactions, background 16292 (homozygous for the BYG15-23 region on chromosome 3 ) had higher fructan concentrations than background 16278. The three-way interaction of background $\times$ ACM033 $\times$ API92 was significant $(P<0.05)$ for sucrose and highly significant for fructan concentrations, indicating that chromosome regions other than those on 5 and 8 may contribute to the low-solids phenotype of AC43. Although other interactions were not significant, most were represented by relatively few families and larger numbers of families would be required for detailed analyses of three-way interactions.

INTERVAL MAPPING. The region on chromosome 5 from API66 to API47 was only significant for DW (Table 3), explaining $36 \%$ and $21 \%$ of the phenotypic variance at 30 and $90 \mathrm{DAH}$, respectively. The BYG15-23 region had an additive effect and increased DW by $0.8 \%$ (Table 3 ). The region on chromosome 8 was significant for DW and fructans at both 30 and 90 DAH. The BYG15-23 region explained $\approx 21 \%$ to $28 \%$ of the variation for DW with additive effects of $0.6 \%$ and $0.9 \%$ at 30 and $90 \mathrm{DAH}$, respectively. This region also explained between $24 \%$ and $69 \%$ of the phenotypic variation for fructans. The additive effect of the BYG15-23 region on chromosome 8 at 30 DAH was $0.6,1.0$, and 4.2 for 1-kestose, neokestose, and total fructan, respectively. These relatively large additive effects on soluble solids are in agreement with previous studies (Havey and Randle, 1996; Lin et al., 1995; Simon, 1995; Wall and Corgan, 1999).

Phenotypic Correlations. Phenotypic correlations for least square means were significant for most traits at 30 and $90 \mathrm{DAH}$ (Table 4). DW was negatively correlated with fructose and positively correlated with sucrose and fructans. Total fructan was highly correlated with 1-kestose and neokestose. Kahane et al. (2001) and McCallum et al. (2006) found similar correlations for DW and fructans in different populations. Fructose was negatively correlated with fructan in agreement with Kahane et al. (2001), Havey et al. (2004), and McCallum et al. (2006). Sucrose was positively correlated with fructans in agreement with Havey et al. (2004) and McCallum et al. (2006). Correlations among DW, sucrose, and fructans were generally lower at $30 \mathrm{~d}$ than $90 \mathrm{~d}$, likely as a result of the hydrolysis of fructans to sucrose and reducing sugars as the onion bulb emerges from dormancy.

\section{Summary}

Onion bulbs showed highly significant phenotypic correlations among DW and concentrations of sucrose, 1-kestose, neokestose, and total fructans at 30 and 90 DAH (Table 4). Regions on chromosomes 5 and 8 are significantly associated with DW and fructan concentrations (Table 1). The lower amounts of soluble carbohydrates in AC43 are conditioned by recessively inherited regions on chromosome 5 and 8 (Table 2). Family background also had a major effect on DW and soluble solids (Tables 1 and 2), ostensibly as a result of a region on chromosome 3 near marker API89 (Galmarini et al., 2001). Interval mapping revealed that the region on chromosome 5 was most significantly associated with increased DW but not with soluble carbohydrates (Table 3 ), in agreement with previous observations (Havey et al., 2004). The region on chromosome 8 was significantly associated with both DW and fructan concentrations (Table 3). McCallum et al. (2006) proposed that a major locus $(F r c)$ in this region on chromosome 8 controls the ability of onion to accumulate fructans. Most onion populations accumulate significant amounts of soluble solids (Foskett and Peterson, 1950) and regions on chromosomes

Table 3. Genetic effects of intervals on chromosomes 5 and 8 significantly associated with percent dry weight (DW) and soluble carbohydrates in segregating families of onion.

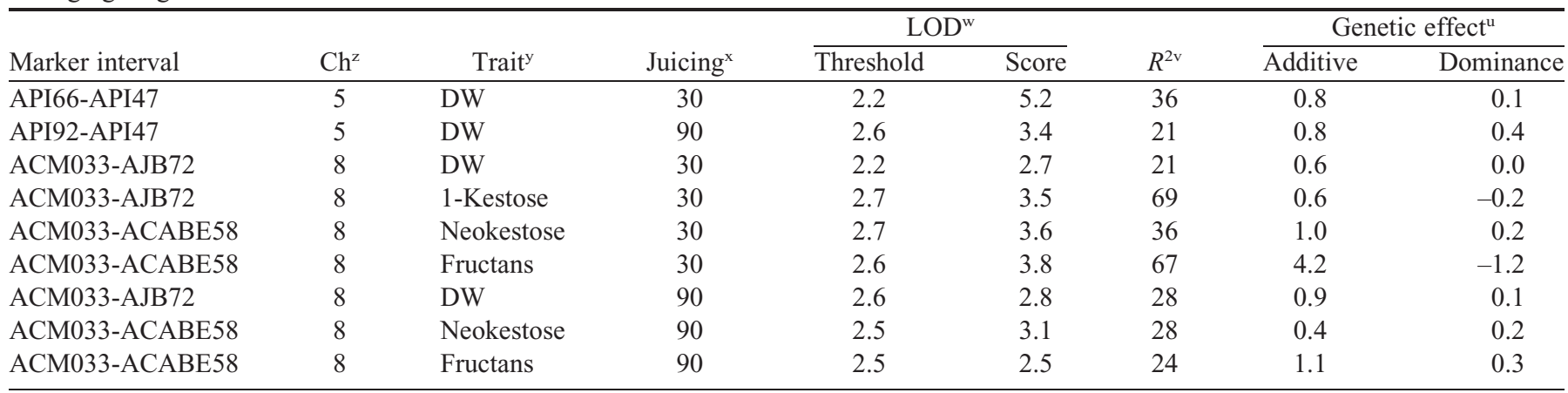

${ }^{\mathrm{z}} \mathrm{Ch}=$ chromosome number.

${ }^{\mathrm{y}} \mathrm{DW}=$ dry weight.

xJuicing of onion bulbs in days after harvest.

${ }^{\mathrm{w}} \mathrm{LOD}=$ logarithm of odds. LOD thresholds were determined by permutation tests conducted 1000 times at 0.05 level (Churchill and Doerge, 1994). Score is the LOD value calculated for the specific genetic interval.

${ }^{\mathrm{v}} R^{2}$ is the percent of phenotypic variation explained by the genetic interval.

udditive and dominance effects of genetic interval were estimated at the highest LOD value. 
Table 4. Significance of phenotypic correlations among least square means of percent dry weight (DW) and soluble carbohydrate concentrations in onion bulbs at 30 and $90 \mathrm{~d}$ after harvest $(\mathrm{DAH})$.

\begin{tabular}{|c|c|c|c|c|c|c|c|}
\hline$\underline{\mathrm{DAH}}$ & Carbohydrate & $\mathrm{DW}^{\mathrm{z}}$ & Fructose & Glucose & Sucrose & 1-Kestose & Neokestose \\
\hline \multirow[t]{5}{*}{30} & Fructose & -0.14 & & & & & \\
\hline & Glucose & 0.08 & $0.87 * * *$ & & & & \\
\hline & Sucrose & $0.47 * * *$ & 0.14 & 0.13 & & & \\
\hline & Neokestose & $0.58 * * *$ & $-0.45^{* * *}$ & -0.18 & $0.51 * * *$ & $0.95 * * *$ & \\
\hline & Fructans & $0.62 * * *$ & $-0.35^{* *}$ & -0.03 & $0.53 * * *$ & $0.98 * * *$ & $0.97 * * *$ \\
\hline \multirow[t]{5}{*}{90} & Fructose & $-0.29 *$ & & & & & \\
\hline & Sucrose & $0.24 *$ & -0.06 & $-0.31 * *$ & & & \\
\hline & 1-Kestose & $0.25^{*}$ & -0.08 & -0.18 & $0.64 * * *$ & & \\
\hline & Neokestose & $0.31 * *$ & 0.02 & -0.14 & $0.45 * * *$ & $0.81 * * *$ & \\
\hline & Fructans & $0.27 *$ & 0.08 & -0.06 & $0.46^{* * *}$ & $0.86^{* * *}$ & $0.96 * * *$ \\
\hline
\end{tabular}

z* $* *, * * *$ represent significance at $P<0.05,0.01,0.001$, respectively.

5 and 8 from BYG15-23 show dominance for increased DW and/or soluble solids (Table 2), indicating that these are likely the wild-type states. The low-solids region on chromosome 5 has been identified only in AC43; low-solids regions on chromosomes 3 and 8 exist in other onions such as the 'Grano' types (McCallum et al., 2006). We propose that onion populations with low solids such as 'Grano' and 'Ailsa Craig' were developed by selecting for recessive regions on chromosomes 3 and 8. 'Ailsa Craig' possesses an additional recessively inherited region on chromosome 5 that is associated with even lower solids than 'Grano' populations.

\section{Literature Cited}

Bark, O.H. and M.J. Havey. 1995. Similarities and relationships among populations of the bulb onion as estimated by nuclear RFLPs. Theor. Appl. Genet. 90:407-414.

Benkeblia, N., S. Onodera, T. Yoshihira, S. Kosaka, and N. Shiomi. 2004. Effect of temperature on soluble invertase activity, and glucose, fructose and sucrose status of onion bulbs (Allium cepa) in store. Intl. J. Food Sci. Nutr. 55:325-331.

Benkeblia, N., N. Shiomi, and M. Osaki. 2007. Kinetics and hydrolysis parameters of total fructooligosaccharides of onion bulbs: Effects of temperature regimes and cultivars. J. Food Biochem. 31:14-27.

Benkeblia, N., K. Ueno, S. Onodera, and N. Shiomi. 2005. Variation of fructooligosaccharides and their metabolizing enzymes in onion bulb (Allium cepa L. cv. Tenshin) during long-term storage. J. Food Sci. 70:S208-S214.

Churchill, G.A. and R.W. Doerge. 1994. Empirical threshold values for quantitative trait mapping. Genetics 138:963-971.

Darbyshire, B. and R.J. Henry. 1978. The distribution of fructans in onions. New Phytol. 81:29-34.

Darbyshire, B. and R.J. Henry. 1979. The association of fructans with high percentage dry weight in onion cultivars suitable for dehydrating. J. Sci. Food Agr. 30:1035-1038.

Darbyshire, B. and R.J. Henry. 1981. Differences in fructan content and synthesis in some Allium species. New Phytol. 87:249-256.

Foskett, R.L. and C.E. Peterson. 1950. Relation of dry matter content to storage quality in some onion varieties and hybrids. Proc. Amer. Soc. Hort. Sci. 55:314-318.

Galmarini, C.R., I.L. Goldman, and M.J. Havey. 2001. Genetic analyses of correlated solids, flavor, and health-enhancing traits in onion (Allium cepa L.). Mol. Genet. Genomics 265:543-551.

Hang, T.T.M., M. Shigyo, S. Yaguchi, N. Yamauchi, and Y. Tashiro. 2004. Effect of single alien chromosome from shallot (Allium cepa L. Aggregatum group) on carbohydrate production in leaf blade of bunching onion (A. fistulosum L.). Genes Genet. Syst. 79:345-350.
Havey, M.J., C.R. Galmarini, A.F. Gokce, and C. Henson. 2004. QTL affecting soluble carbohydrate concentrations in stored onion bulbs and their association with flavor and health-enhancing attributes. Genome 47:463-468.

Havey, M.J. and W.M. Randle. 1996. Combining abilities for yield and bulb quality among long- and intermediate-day open-pollinated onion populations. J. Amer. Soc. Hort. Sci. 121:604-608.

Henson, C.A. and D.P. Livingston III. 1996. Purification and characterization of an oat fructan exohydrolase that preferentially hydrolyzes [beta]-2,6-fructans. Plant Physiol. 110:639-644.

Jaime, L., M.A. Martin-Cabrejas, E. Molla, F.J. Lopez-Andreu, and R.M. Esteban. 2001. Effect of storage on fructan and fructooligosaccharide of onion (Allium cepa L.). J. Agr. Food Chem. 49:982988.

Jakse, J., W. Martin, J. McCallum, and M. Havey. 2005. Single nucleotide polymorphisms, indels, and simple sequence repeats for onion cultivar identification. J. Amer. Soc. Hort. Sci. 130:912-917.

Kahane, R., E. Vialle-Guérin, I. Boukema, D. Tzanoudakis, C. Bellamy, C. Chamaux, and C. Kik. 2001. Changes in non-structural carbohydrate composition during bulbing in sweet and high-solid onions in field experiments. Environ. Exp. Bot. 45:73-83.

King, J.J., J.M. Bradeen, O. Bark, J.A. McCallum, and M.J. Havey. 1998. A low-density genetic map of onion reveals a role for tandem duplication in the evolution of an extremely large diploid genome. Theor. Appl. Genet. 96:52-62.

Lin, M., J.F. Watson, and J.R. Baggett. 1995. Inheritance of soluble solids and pyruvic acid content of bulb onions. J. Amer. Soc. Hort. Sci. 120:119-122.

Martin, W., J. McCallum, M. Shigyo, J. Jakse, J. Kuhl, N. Yamane, M. Pither-Joyce, A. Gokce, K. Sink, C. Town, and M. Havey. 2005. Genetic mapping of expressed sequences in onion and in silico comparisons with rice show scant colinearity. Mol. Genet. Genomics 274:197-204.

Masuzaki, S., S. Yahuchi, N. Yamauchi, and M. Shigyo. 2007. Morphological characterisation of multiple alien addition lines of Allium reveals the chromosomal locations of gene(s) related to bulb formation in Allium cepa L. J. Hort. Sci. Biotechnol. 82:393-396.

McCallum, J., A. Clarke, M. Pither-Joyce, M. Shaw, R. Butler, D. Brash, J. Scheffer, I. Sims, S. van Heusden, M. Shigyo, and M.J. Havey. 2006. Genetic mapping of a major gene affecting onion bulb fructan content. Theor. Appl. Genet. 112:958-967.

Moshfegh, A.J., J.E. Friday, J.P. Goldman, and J.K.C. Ahuja. 1999. Presence of inulin and oligofructose in the diets of Americans. J. Nutr. 129:1407S-1411S.

Randle, W. 1992. Sampling procedures to estimate flavor potential in onion. HortScience 27:1116-1117.

Ritsema, T., J. Joling, and S. Smeekens. 2003. Patterns of fructan synthesized by onion fructan:fructan 6G-fructosyltransferase 
expressed in tobacco BY2 cells-Is fructan:fructan 1-fructosyltransferase needed in onion? New Phytol. 160:61-67.

Ritsema, T. and S. Smeekens. 2003. Fructans: Beneficial for plants and humans. Curr. Opin. Plant Biol. 6:223-230.

Roberfroid, M.B. 2005. Introducing inulin-type fructans. Brit. J. Nutr. 93:S13-S25.

Shiomi, N. 1989. Properties of fructosyltransferases involved in the synthesis of fructan in liliaceous plants. J. Plant Physiol. 134:151155.

Shiomi, N., S. Onodera, and H. Sakai. 1997. Fructo-oligosaccharide content and fructosyltransferase activity during growth of onion bulbs. New Phytol. 136:105-113.

Simon, P.W. 1995. Genetic analysis of pungency and soluble solids in long-storage onions. Euphytica 82:1-8.

Sinclair, P.J., R.J. Neeson, and E.W.R. Barlow. 1995. Osmotic potential and soluble solids concentration in onion (Allium cepa) bulbs. J. Sci. Food Agr. 69:211-214.
Suzuki, M. and J.A. Cutcliffe. 1989. Fructans in onion bulbs in relation to storage life. Can. J. Plant Sci. 69:1327-1333.

Vijn, I., A. van Dijken, N. Sprenger, K. van Dun, P. Weisbeek, A. Wiemken, and S. Smeekens. 1997. Fructan of the inulin neoseries is synthesized in transgenic chicory plants (Cichorium intybus L.) harbouring onion (Allium cepa L.) fructan:fructan 6G- fructosyltransferase. Plant J. 11:387-398.

Wall, A. and J. Corgan. 1999. Heritability estimates and progeny testing of phenotypic selections for soluble solids content in dehydrator onion. Euphytica 106:7-13.

Wang, C., W. Van den Ende, and J. Tillberg. 2000. Fructan accumulation induced by nitrogen deficiency in barley leaves correlates with the level of sucrose:fructan 6-fructosyltransferase mRNA. Planta 211:701-707.

Yasin, H.J. and G. Bufler. 2007. Dormancy and sprouting in onion (Allium cepa L.) bulbs. I. Changes in carbohydrate metabolism. J. Hort. Sci. Biotechnol. 82:89-96. 